Proyecciones Journal of Mathematics

Vol. 41, $\mathrm{N}^{\circ}$ 1, pp. 101-110, February 2022.

Universidad Católica del Norte

Antofagasta - Chile

\title{
Total neighborhood prime labeling of some trees
}

\author{
T. J. Rajesh Kumar
}

TKM College of Engineering, India

Received : March 2021. Accepted : September 2021

\begin{abstract}
Let $G$ be a graph with $p$ vertices and $q$ edges. A total neighborhood prime labeling of $G$ is a labeling in which the vertices and edges are assigned labels from 1 to $p+q$ such that the gcd of labeling in the neighborhood of each non degree 1 vertex is equal to 1 and the gcd of labeling in the edges of each non degree 1 vertex is equal to 1 . A graph that admits a total neighborhood prime labeling is called a total neighborhood prime graph. In this paper, we examine total neighborhood prime labeling of trees such as $(n, k, m)$ double star trees, spiders, caterpillars and firecrackers.
\end{abstract}

Keywords: Total Neighborhood prime labeling, Double star trees, Spiders, Caterpillars, Firecrackers.

Subjclass [2000]: $05 \mathrm{C} 78$. 


\section{Introduction}

Graphs considered here are simple, finite, connected and undirected graphs and follow [2] for basic notations and terminology of graph theory. The concept of prime labeling was developed by Roger Entriger and first introduced by Tout, Dabbacey, and Howalla [8]. A prime labeling on a simple graph $G$ with $n$ vertices in the vertex set $V(G)$ is an assignment of the integers 1 to $n$ as labels of the vertices such that each pair of labels from adjacent vertices is relatively prime. A graph admits such a labeling is called a prime graph. Over the last thirty-eight years, prime labeling have been developed or labeling have been shown to well known classes of graphs. A detailed list of graphs that have been proven to be prime was shown in Gallian's dynamic survey of graph labeling [1].

The concept of a neighborhood prime labeling of a graph is a variation of a prime labeling which involves the neighborhood of a vertex $v$. This is the set of all vertices in $G$ that are adjacent to $v$ and is denoted by $N(v)$ or $N_{G}(v)$. A neighborhood prime labeling of a graph $G$ with $n$ vertices is a labeling of the vertex set with the integers 1 to $n$ in which for each vertex $v \in V(G)$ of degree greater than 1, the gcd of the labels of the vertices in $N(v)$ is one. A graph which admits a neighborhood prime labeling is called a neighborhood prime graph. The neighborhood prime labeling was first introduced and developed by Patel and Shrimali [3, 4]. They proved all paths, complete graphs, wheels, helms, flowers, grid graphs and union of paths and wheels are neighborhood prime.

In [6], Rajesh Kumar and Mathew Varkey extended the neighborhood prime labeling to total neighborhood prime labeling by labeling the edges. A graph $G$ on $p$ vertices and $q$ edges is said to have a total neighborhood prime labeling if there exists a labeling from the vertices and edges of $G$ to the first $p+q$ natural numbers such that for each vertex in $G$ of degree at least two, the gcd of labeling on its neighborhood vertices is one and for each vertex of degree at least two the gcd of labeling on its incident edges is one. The total neighborhood prime labeling of complete graphs, star graphs, wheels, Fan graphs, Paths, Comb graphs and cycles are discussed in [6]. Shrimali and Pandiya [7], proved disjoint union of paths $P_{m} U P_{n}$, disjoint union of sunlet graphs $S_{n} U S_{m}$, disjoint union of wheel graphs $W_{n} U W_{n}$ and the subdivision of bistar graph are total neighborhood prime. In this paper, considered the graphs $(n, k, m)$ double star trees, spiders, caterpillars, firecrackers and their total neighborhood prime labeling.

Definition 1.1. Let $n, k, m$ be integers with $k \leq m$. The $(n, k, m)$ double 
star tree, $D S_{n, k, m}$ is the graph with $V\left(D S_{n, k, m}\right)=\left\{v_{1}, v_{2}, \ldots v_{n}, v_{n+1}, \ldots v_{n+k-1}, v_{n+k} \ldots\right.$, $\left.v_{n+k+m-2}\right\}$ and $E\left(D S_{n, k, m}\right)=\left\{v_{j} v_{j+1}: 1 \leq j \leq n-1, v_{1} v_{n+j}: 1 \leq j \leq\right.$ $\left.k-1: v_{n} v_{n+k+j}: 0 \leq j \leq m-2\right\}$ In the $(n, k, m)$ double star tree we have a path of length $n$ whose end vertices $v_{1}$ and $v_{n}$ are the central vertices for a $k$-star and a $m$-star respectively.

Definition 1.2. A spider graph is a tree with one vertex of degree at least 3 and all others with degree at most 2. A spider can also be viewed as a collection of paths $P_{n_{1}}, P_{n_{2}}, \ldots, P_{n_{k}}$ with one end of each path adjoined to a central vertex.

Definition 1.3. A caterpillar is a tree in which all the vertices are within distance 1 of a central path. Caterpillars can be constructed from a path $P_{n}$ in which each of the $n-2$ interior vertices either remains as degree 2 or is adjacent to at least one pendent vertex.

Definition 1.4. An $(n, k)$ firecracker tree, denoted by $F_{n, k}$ is a path $P_{n}$ in which each vertex is also a leaf on a $k$-star.

\section{Main Results}

The total neighbourhood prime labeling of $(n, k, m)$ double star tree, Spiders, Caterpillars and Firecracker trees are considered in this section.

Theorem 2.1. Any $(n, k, m)$ double star tree has a total neighborhood prime labeling.

Proof. The vertices in the path of $(n, k, m)$ double star tree are denoted by $v_{1}, v_{2}, \ldots, v_{n}$, the vertices adjacent to $v_{1}$ are denoted by $v_{n+1}, v_{n+2}, \ldots, v_{n+k-1}$ and the vertices adjacent to $v_{n}$ are $v_{n+k}, v_{n+k+1}, \ldots, v_{n+k+m-2}$. The edge set is $\left\{v_{j} v_{j+1}: 1 \leq j \leq n-1, v_{1} v_{n+j}: 1 \leq j \leq k-1, v_{n} v_{n+k+j}: 0 \leq j \leq\right.$ $m-2\}$.

First label the vertices of the path using the function $f$ define from $\left\{v_{1}, v_{2}, \ldots, v_{n}\right\}$ to $\{1,2,3, \ldots, n\}$ as

Case 1: If $n$ is odd

$$
\begin{array}{r}
f\left(v_{2 i-1}\right)=\frac{n+2 i-1}{2}, \quad 1 \leq i \leq \frac{n+1}{2} \\
f\left(v_{2 i}\right)=i, \quad 1 \leq i \leq \frac{n-1}{2}
\end{array}
$$


Case 2: If $n$ is even

$$
\begin{array}{r}
f\left(v_{2 i-1}\right)=\frac{n+2 i}{2}, \quad 1 \leq i \leq \frac{n}{2} \\
f\left(v_{2 i}\right)=i, \quad 1 \leq i \leq \frac{n}{2},
\end{array}
$$

Now label the $k-1$ vertices adjacent to $v_{1}$ with the integers $n+1, n+$ $2, \ldots, n+k-1$ arbitrarily. Label the remaining vertices adjacent to $v_{n}$ arbitrarily with the remaining integers $n+k, n+k+1, \ldots, n+k+m-2$.

The edges of the path $\left\{v_{j} v_{j+1}, 1 \leq j \leq n-1\right\}$ are labeled using the function $g$ defined from edge set to $\{n+k+m-2+j, 1 \leq j \leq n-1\}$ as $g\left(v_{j} v_{j+1}\right)=n+k+m-2+j, 1 \leq j \leq n-1$. Now label the $k-1$ edges starting from $v_{1}$ with $2 n+k+m-2,2 n+k+m-1, \ldots, 2 n+2 k+m-4$ arbitrarily. Label the remaining $m-1$ edges starting from $v_{n}$ arbitrarily with the remaining integers $2 n+2 k+m-3,2 n+2 k+m-2, \ldots, 2 n+2 k+2 m-5$.

Let $v$ be an arbitrary vertex in $(n, k, m)$ double star tree with degree greater than one. We consider the following two cases for this vertex $v$ to demonstrate the labeling is total neighborhood prime.

Case (1) If $v=v_{1}$ and $v_{n}$, the neighborhood vertices are labeled with consecutive integers and the incident edges are labeled with consecutive integers. So the gcd of labeling on the neighborhood vertices of $v$ is one and the gcd of labeling on the incident edges of $v$ is one.

Case (2) If $v=v_{i}$ for $i=2,3, \ldots, n-1$, the neighborhood vertices of $v$ are labeled with consecutive integers and the edges incident in $v$ are labeled with consecutive integers.

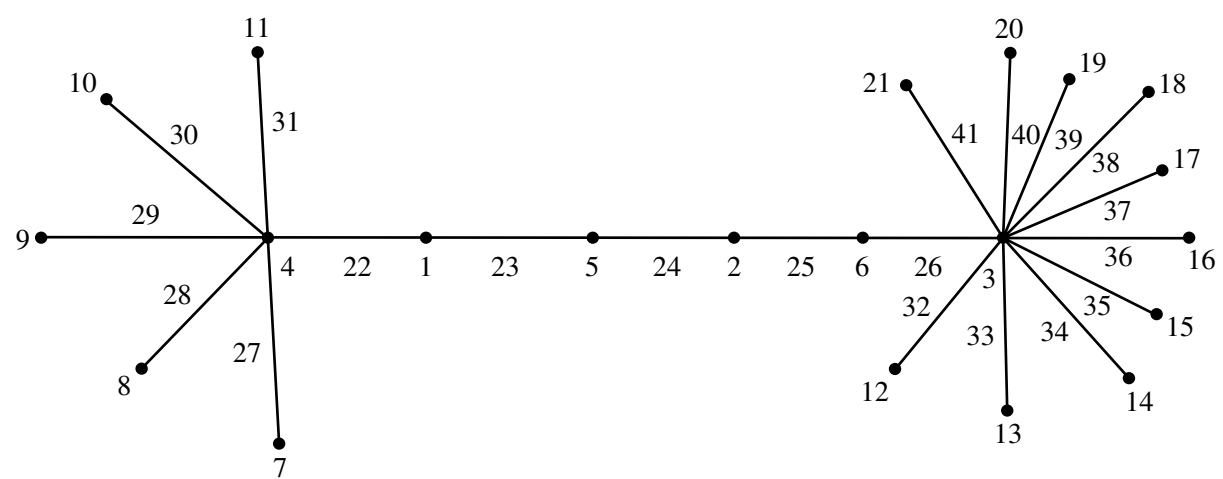

Figure 1: A total neighborhood prime labeling of $(6,6,11)$ double star tree. 
Theorem 2.2. All spiders are total neighborhood prime.

Proof. A spider can be viewed as a collection of paths $P_{n_{1}}, P_{n_{2}}, \ldots, P_{n_{k}}$ with one end of each path adjoined to a central vertex $v_{0}$. We label the central vertex $v_{0}$ of the spider by assigning the label 1 . For each path $P_{n_{j}}$, we consider the vertices as $v_{n_{j}}^{1}, v_{n_{j}}^{2}, \ldots, v_{n_{j}}^{m}$ where $m=n_{j}$ with $v_{n_{j}}^{1}$ being adjacent to the central vertex $v_{0}$ and $v_{n_{j}}^{m}$ as the leaf.

For each path consider the labeling function as

$$
f_{N}\left(v_{n_{j}}^{i}\right)= \begin{cases}N+\frac{i+1}{2}, & \text { if } i \text { is odd } \\ N+\left\lfloor\frac{m}{2}\right\rfloor+\frac{i}{2}, & \text { if } i \text { is even }\end{cases}
$$

where $N=1+n_{1}+n_{2}+\cdots+n_{j-1}$. In each path, we assign smallest label at the first vertex and shift the label to begin each path with the smallest available label. If every length of the path $n_{j}$ are even, label the last path by swapping the labels of $f_{N}\left(v_{n_{j}}^{n_{j}-i+1}\right)$ and $f_{N}\left(v_{n_{j}}^{i}\right)$ for each $i=1,2, \ldots, n_{j}$.

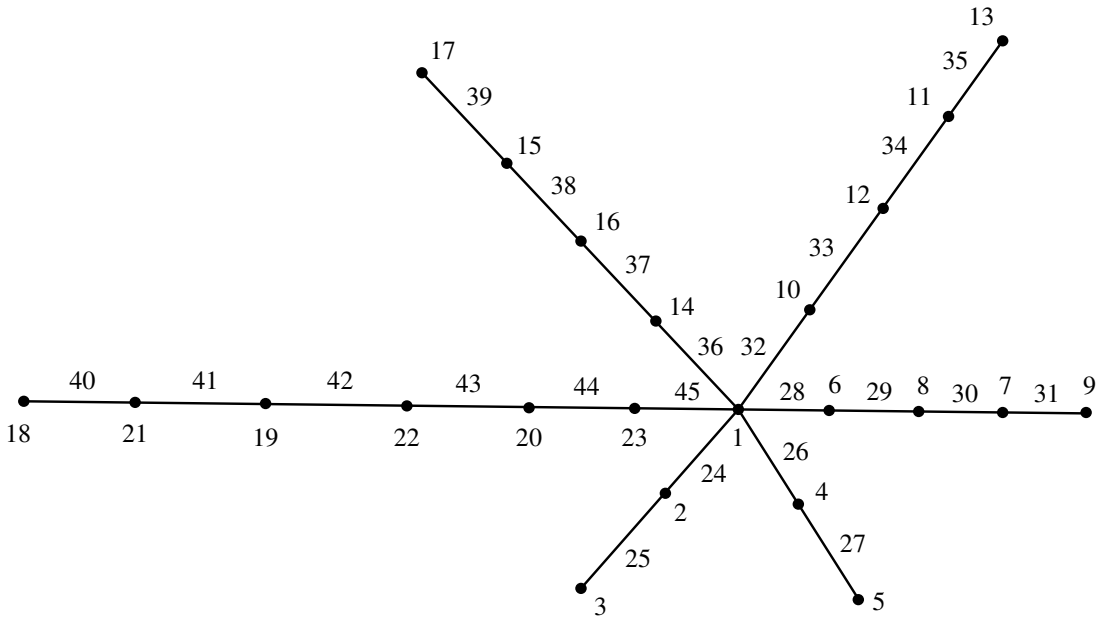

Figure 2: Total neighborhood prime labeling of a spider with paths

$$
P_{2}, P_{2}, P_{4}, P_{4}, P_{4} \text { and } P_{6} \text {. }
$$

Consider the labeling for the edges $e_{n_{j}}^{i}=v_{n_{j}}^{i-1} v_{n_{j}}^{i}$ where $v_{n_{j}}^{0}=v_{0}$ as $f_{N}\left(e_{n_{j}}^{i}\right)=M+N+(i-1), 1 \leq i \leq n_{j}$. Here $N=1+n_{1}+n_{2}+\cdots+n_{j-1}$ and $M$ is one above the sum of lengths of all the paths. In each path assign smallest label in the first edge and shift the label to begin each path with the smallest available label. If every length of the path $n_{j}$ are even, label 
the last path by swapping the labels of $f_{N}\left(e_{n_{j}}^{n_{j}-i+1}\right)$ and $f_{N}\left(e_{n_{j}}^{i}\right)$ for each $i=1,2, \ldots, n_{j}$.

Consider a vertex $v$ with degree 2 . If $v$ is on the path $P_{n}$ and is adjacent to the central vertex, then $f_{N}(v)$ contains the label 1 and the labeling on the incident edges are consecutive integers. If $v$ is on the path, but not adjacent to the central vertex, the neighborhood vertices are labeled by consecutive integers and the edges incident on $v$ are labeled by consecutive integers. Finally consider $v$ is the central vertex. The label on the first vertex of $P_{n_{1}}$, is always 2 . If the length of any of the paths is odd, then take $P_{n_{1}}$ to be a path of odd length. In this way, the first vertex of the next path is labeled by an odd integer. If none of the lengths are odd, then we use the swapping on the last path $P_{n_{k}}$ and its first vertex attached on the central vertex is labeled by an odd integer. If the length of any of the path is odd, the gcd of labeling on the edges incident on $v$ is one. If none of the length are odd, then using the above swapping and the gcd of labeling on the edges incident on $v$ is one. This proves the spider is total neighborhood prime.

Now, we consider one lemma which says a way in a total neighborhood prime graph to add a pendent edge or an edge connecting to a leaf and maintain a total neighborhood prime labeling. Use this lemma for labeling the caterpillars and firecrackers.

Lemma 2.1. Assume $G$ is a total neighborhood prime graph with $p$ vertices and $q$ edges. Let $v$ be a vertex in $G$ with $\operatorname{deg}(v)>1$. The graph $G^{\prime}$ obtained by attaching a pendent edge to the vertex $v$ is total neighborhood prime.

Proof. Let $f: V \cup E \rightarrow\{1,2, \ldots, p+q\}$ be the total neighborhood prime labeling for $G$. Let $v^{\prime}$ be the new vertex adjacent to $v$, then $V\left(G^{\prime}\right)=$ $V(G) \cup\left\{v^{\prime}\right\}$ and $E\left(G^{\prime}\right)=E(G) \cup\left\{v v^{\prime}\right\}$, where $v v^{\prime}$ is the edge connecting the vertex $v$ to $v^{\prime}$. Define a function $g: V\left(G^{\prime}\right) \cup E\left(G^{\prime}\right) \rightarrow\{1,2, \ldots, p+$ $q, p+q+1, p+q+2\}$ by assigning $g\left(v^{\prime}\right)=p+q+1, g\left(v v^{\prime}\right)=p+q+2$, $g(v)=f(v)$ for all $v \in V(G)$ and $g(u v)=f(u v)$ for all edge $u v \in E(G)$.

Let $u$ be a vertex in $G^{\prime}$ with $\operatorname{deg}(u)>1$, which is not $v^{\prime}$ since it is a pendent vertex. For the case of $u=v$, we have $N_{G^{\prime}}(v)=N_{G}(v) \cup\left\{v^{\prime}\right\}$. Since $N_{G}(v) \subset N_{G^{\prime}}(v)$ and gcd of labeling on the neighborhood vertices of $v$ in $G$ is one, the gcd of labeling on the neighborhood vertices of $v$ in $G^{\prime}$ is one. Also the gcd of labeling on the incident edges of $v$ in $G^{\prime}$ is one. If $u \neq v, N_{G^{\prime}}(u)=N_{G}(u)$. Since $f$ is a total neighborhood prime labeling, $g$ is 
also a total neighborhood prime labeling. Thus $G^{\prime}$ is a total neighborhood prime graph.

Theorem 2.3. All caterpillars have total neighborhood prime labeling.

\section{Proof:}

Consider a caterpillar with a central path $P_{n}$. First label the path using the path labeling given in [6].

Let $\dot{v}_{1}, v_{2}, \ldots v_{n}$ are the consecutive vertices of path $P_{n}$ and $e_{i}=v_{i} v_{i+1}$, $1 \leq i \leq n-1$ are the corresponding edges. The mapping $f: V U E \rightarrow$ $\{1,2,3, \ldots, 2 n-1\}$ defined by

Case (i): If $n$ is odd.

$$
\begin{aligned}
f\left(v_{2 i-1}\right) & =\frac{n+2 i-1}{2}, 1 \leq i \leq \frac{n+1}{2} \\
f\left(v_{2 i}\right) & =i, 1 \leq i \leq \frac{n-1}{2} \\
f\left(e_{i}\right) & =n+i, 1 \leq i \leq n-1
\end{aligned}
$$

Case (2): If $n$ is even

$$
\begin{aligned}
f\left(v_{2 i-1}\right) & =\frac{n+2 i}{2}, 1 \leq i \leq \frac{n}{2} \\
f\left(v_{2 i}\right) & =i, \quad 1 \leq i \leq \frac{n}{2} \\
f\left(e_{i}\right) & =n+i, \quad 1 \leq i \leq n-1
\end{aligned}
$$

Then since the interior path vertices were initially degree 2 , the above lemma provides a way to assign labels to each pendent vertex while maintaining the total neighborhood prime labeling condition at each step. 


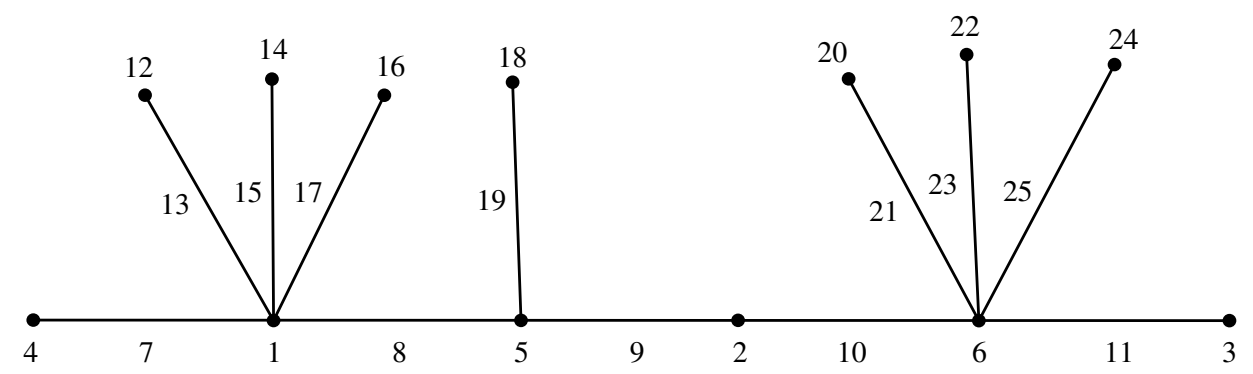

Figure 3: Total neighborhood prime labeling of a caterpillar.

Theorem 2.4. The firecracker tree $F_{n, k}$ has a total neighborhood prime labeling for all $n \geq 1, k \geq 1$.

\section{Proof:}

The firecracker tree is a path if $k=1$ and a caterpillar if $k=2$. The path and caterpillar are total neighborhood prime. Now consider the case of $k=3$, the vertices along the path are denoted by $v_{1}, v_{2}, \ldots, v_{n}$, the vertices adjacent to the path are denoted by $u_{1}, u_{2}, \ldots, u_{n}$ and the leaves are denoted by $w_{1}, w_{2}, \ldots, w_{n}$ where $N\left(u_{i}\right)=\left\{v_{i}, w_{i}\right) .1 \leq i \leq n$. The edges are denoted by $\left\{v_{i} u_{i}, u_{i} w_{i}, 1 \leq i \leq n\right\} \cup\left\{v_{i} v_{i+1}, 1 \leq i \leq n-1\right\}$. We assign labels using a function $f: V \cup E\left(F_{n, 3}\right) \rightarrow\{1,2, \ldots, 3 n, 3 n+$ $1,3 n+2, \ldots, 6 n+1\}$. The path vertices $v_{1}, v_{2}, \ldots, v_{n}$ are labeled using the vertex labeling in theorem (2.3). Now label the $n^{\text {th }}$ vertex $u_{n}$ adjacent to the vertex $v_{n}$ as $f\left(u_{n}\right)=p$ where $p$ is a prime with $n+1 \leq p \leq 2 n$ using Bertrand's Postulate from Prime Number Theorem.

For the vertices $u_{1}, u_{2}, \ldots u_{n-1}$, assign the labels $\{n+1, n+2, \ldots, 2 n\} \backslash\{p\}$ in any order. Finally, label the vertices $w_{i}$, using the co prime mapping function $g$ from Pomerance and Selfridge's theorem [5] . Using the theorem, since $\{2 n+1,2 n+2, \ldots, 3 n\}$ are consecutive integers there exists a function $g$ such that $f\left(w_{i}\right)=g\left(f\left(u_{i}\right)\right), 1 \leq i \leq n$. So the vertices of $F_{n, 3}$ are labeled with first $3 n$ natural numbers. The edges are labelling in the following order. The edge $w_{1} u_{1}$ labeled as $3 n+1, u_{1} v_{1}$ labeled as $3 n+2$, $v_{1} v_{2}$ labeled as $3 n+3, w_{2} u_{2}$ labeled as $3 n+4, u_{2} v_{2}$ labeled as $3 n+5, v_{2} v_{3}$ labeled $3 n+6$ and so on. 
We consider the following cases for demonstrate this labeling is neighborhood prime. Let $v$ be an arbitrary vertex in $F_{n, 3}$ with degree greater than 1.

Case (1): If $v=v_{1}$, then $N(v)=\left\{u_{1}, v_{2}\right\}$, since the second vertex of any path is labeled by 1 using the labeling in theorem $(2.3), f\left(v_{2}\right)=1$. So the gcd of the labeling on the neighborhood vertices of $v_{1}$ is one. Also the labeling on the edges incident with $v_{1}$ are $\{3 n+2,3 n+3\}$ and the gcd is one.

Case (2): When $v$ is an interior path vertex, i.e $v=v_{i} ; i=2,3, \ldots, n-1$. The neighborhood vertices of $v$ in path $P_{n}$ is a subset of the neighborhood vertices of $v$ in $F_{n, 3}$ and the labeling on the path $P_{n}$ is a total neighborhood prime labeling. So the gcd of labeling on the neighborhood vertices of $v$ in $F_{n, 3}$ is same as the gcd of labeling on the neighborhood vertices of $v$ in $P_{n}$. Since gcd $\left\{f\left(N_{P_{n}}(v)\right)\right\}=1$, the labeling is total neighborhood prime.

Case (3): If $v=v_{n}$, then $N(v)=\left\{v_{n-1}, u_{n}\right\}$. Since $f\left(u_{n}\right)$ was chosen to be prime $p>n \geq f\left(v_{n-1}\right)$, the gcd of these two labels is one. Also the labeling on the edges incident with $v_{n}$ are consecutive integers and the gcd is one.

Case (4): If $v=u_{i}$, for some $i=1,2, \ldots, n, N(v)=\left\{v_{i}, w_{i}\right\}$. By the co prime mapping theorem in [5] gcd $\left\{f\left(v_{i}\right), f\left(w_{i}\right)\right\}=\operatorname{gcd}\left\{f\left(u_{i}\right), g\left(f\left(u_{i}\right)\right)\right\}=$ 1. Also the labeling on the edges incident with $u_{i}$ are consecutive integers and the gcd is one. So $F_{n, 3}$ is a total neighborhood prime graph.

Now create $F_{n, k}, k>3$ from $F_{n, 3}$ by attaching $k-3$ pendent vertices to each vertex $u_{1}, u_{2}, \ldots, u_{n}$. Then using lemma (2.1) $F_{n, k}$ is a total neighborhood prime graph for all $k \geq 3$.

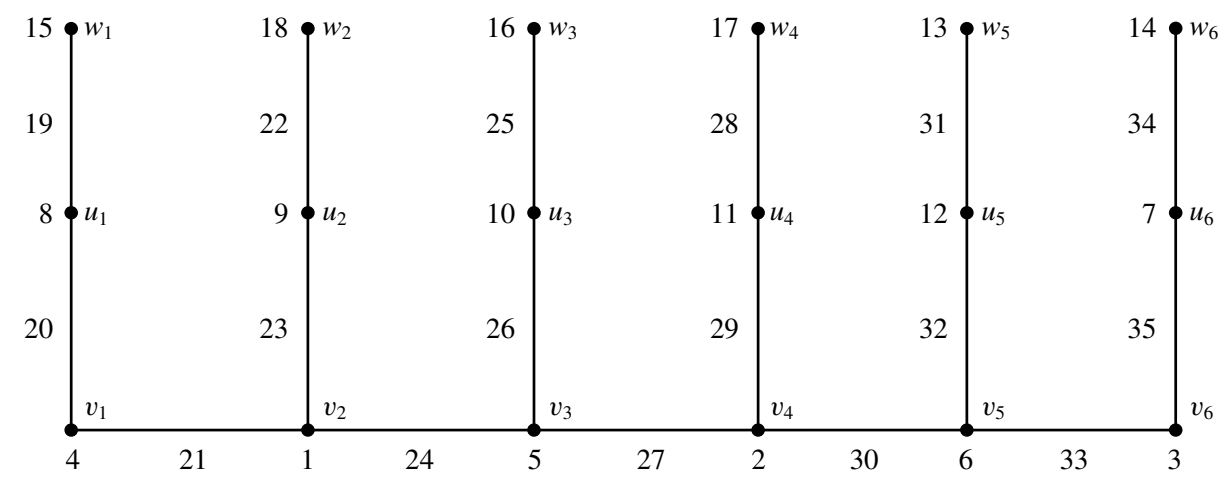

Figure 4: Total neighborhood prime labeling of the firecracker $F_{6,3}$. 


\section{References}

[1] J. A. Gallian, "A dynamic survey of graph labeling", The electronic journal of combinatorics, \#DS6, 2019.

[2] F. Harary, Graph theory. Boston: Addison Wesley, 1972.

[3] S. K. Patel and N. P. Shrimali, "Neighborhood prime labeling", International Journal of Mathematics and Soft Computing, vol. 5, no. 2, pp. 135-143, 2015. [On line]. Available: https:/ / bit.ly/ 33oIfVF

[4] S. K. Patel and N. P. Shrimali, "Neighborhood-prime labeling of some union graphs", International journal of mathematics and soft computing, vol. 6, no. 1, pp. 39-47, 2016. doi: 10.26708/ijmsc.2016.1.6.04

[5] C. Pomerance and J. L. Selfridge, "Proof of D. J. Newmans coprime mapping conjecture", Mathematika, vol. 27, no. 1, pp. 69-83, 1980. doi: 10.1112/ s0025579300009955

[6] T. J. Rajesh Kumar and T. K. Mathew Varkey, "A Note on total neighborhood prime labeling", International journal of pure and applied mathematics, vol.118, no. 4, pp. 1007-1013, 2018. [On line]. Available: https:// bit.ly/ 3HTJmLR

[7] N. P. Shrimali and P. B. Pandya, "Total neighborhood prime labeling of some graphs", International journal of scientific research in mathematical and statistical sciences, vol. 5, no. 6, pp. 157-163, 2018. [On line]. Available: https:/ / bit.ly/ 3rcscCk

[8] A. Tout, A. N. Dabboucy and K. Howalla, "Prime labeling of graphs", National Academy Science Letters, vol. 11, pp. 365-368, 1982.

\section{T.J. Rajesh Kumar}

Department of Mathematics, TKM College of Engineering, Kollam, Kerala, India

e-mail: vptjrk@gmail.com 